\title{
Some relationships between left (right) semi-uninorms and implications on a complete lattice
}

\author{
Yuan Wang ${ }^{1,}$, Keming Tang ${ }^{1}$, Zhudeng Wang ${ }^{2}$ \\ ${ }^{1}$ College of Information Science and Technology, Yancheng Teachers University, Yancheng 224002, People's Republic of China \\ ${ }^{2}$ School of Mathematical Sciences, Yancheng Teachers University, Jiangsu 224002, People's Republic of China \\ Email address: \\ yctuwangyuan@163.com (Yuan Wang),tkmchina@126.com (Keming Tang), zhudengwang2004@163.com (Zhudeng Wang)
}

\section{To cite this article:}

Yuan Wang, Keming Tang, Zhudeng Wang. Some Relationships between Left (Right) Semi-Uninorms and Implications on a Complete Lattice. Automation, Control and Intelligent Systems. Vol. 2, No. 3, 2014, pp. 33-41. doi: 10.11648/j.acis.20140203.12

\begin{abstract}
In this paper, we study the relationships between left (right) semi-uninorms and implications on a complete lattice. We firstly discuss the residual operations of left and right semi-uninorms and show that the right (left) residual operator of a conjunctive right (left) $\vee$-distributive left (right) semi-uninorm is a right $\wedge$-distributive implication that satisfies the neutrality principle. Then, we investigate the left and right semi-uninorms induced by an implication, give some conditions such that two operations induced by an implication constitute left or right semi-uninorms, and demonstrate that the operations induced by a right $\wedge$-distributive implication, which satisfies the order property or neutrality principle, are left (right) $\vee$-distributive left (right) semi-uninorms or right (left) semi-uninorms. Finally, we reveal the relationships between conjunctive right (left) $\vee$-distributive left (right) semi-uninorms and right $\wedge$-distributive implications which satisfy the neutrality principle.
\end{abstract}

Keywords: Fuzzy Logic, Fuzzy Connective, Left (Right) Semi-Uninorm, Implication, Neutrality Principle, Order Property

\section{Introduction}

In fuzzy logic, the truth function for a conjunction connective is usually taken as a triangular norm [1] $(t$-norm for short), which is monotone, associative, commutative and has neutral element 1 . However, the $t$-norms cannot deal with natural interpretations of linguistic words because the axioms of $t$-norms are strong. To interpret the noncommutative conjunctions, Flondor et al. [2] introduced non-commutative $t$-norms by throwing away the axiom of commutativity of $t$-norms and using them to construct pseudo- $B L$-algebras. About another axiom of $t$-norms, associativity, Fodor and Keresztfalvi [3] underlined that "if one works with binary conjunctions and there is no need to extend them for three or more arguments, as happens, for example, in the inference pattern called generalized modus ponens, associativity of the conjunction is an unnecessarily restrictive condition". By removing the associativity and commutativity from the axioms of $t$ - norms, weak $t$-norms [4] and pseudo-t-norms [5] were introduced and discussed.

Uninorms, introduced by Yager and Rybalov [6] and studied by Fodor et al. [7], are special aggregation oper- ators that have proven useful in many fields like fuzzy logic, expert systems, neural networks, aggregation, and fuzzy system modeling [8-11]. This kind of operation is an important generalization of both $t$-norms and $t$-conorms and a special combination of $t$-norms and $t$-conorms [7]. However, there are real-life situations when truth functions cannot be associative or commutative. By throwing away the commutativity from the axioms of uninorms, Mas et al. introduced the concepts of left and right uninorms on $[0,1]$ in [12] and later in a finite chain in [13], Wang and Fang [14-15] studied the left and right uninorms on a complete lattice. By removing the associativity and commutativity from the axioms of uninorms, Liu [16] introduced the concept of semi-uninorms and $\mathrm{Su}$ et al. [17] discussed the notion of left and right semi-uninorms on a complete lattice. On the other hand, it is well known that a uninorm (semiuninorm, left and right uninorms) $U$ can be conjunctive or disjunctive whenever $U(0,1)=0$ or 1 , respectively. This fact allows to use uninorms in defining fuzzy implications and coimplications [14-16, 18-20].

In this paper, based on $[14,16,18-20]$, we study left (right) semi-uninorms and implications on a complete lattice. After recalling some necessary definitions and 
examples about the left and right semi-uninorms on a complete lattice in Section 2, we discuss the residual operations of left and right semi-uninorms in Section 3 and show that the right (left) residual operator of a conjunctive right (left) $\vee$-distributive left (right) semi-uninorm is a right $\wedge$-distributive implication that satisfies the neutrality principle. In Section 4, we investigate the left and right semi-uninorms induced by an implication and give some conditions such that the operations induced by an implication become either left or right semi-uninorms. In Section 5 , we reveal the relationships between conjunctive right (left) $\vee$-distributive left (right) semi-uninorms and right $\wedge$-distributive implications which satisfy the neutrality principle.

The knowledge about lattices required in this paper can be found in [21].

Throughout this paper, unless otherwise stated, $L$ always represents any given complete lattice with maximal element 1 and minimal element $0 ; J$ stands for any index set.

\section{Left and Right Semi-Uninorms}

Noting that the commutativity and associativity are not desired for aggregation operators in a number of cases, Liu [16] introduced the concept of semi-uninorms and Su et al. [17] studied the notions of left and right semi-uninorms. Here, we recall some necessary definitions and examples of the left and right semi- uninorms on a complete lattice.

Definition 2.1 (Su et al. [17]). A binary operation $U$ on $L$ is called a left (right) semi-uninorm if it satisfies the following two conditions:

(U1) there exists a left (right) neutral element, i.e., an element $e_{L} \in L \quad\left(e_{R} \in L\right)$ satisfying $U\left(e_{L}, x\right)=x$ $\left(U\left(e_{R}, x\right)=x\right.$ for all $x \in L$,

(U2) $U$ is non-decreasing in each variable.

For any left (right) semi-uninorm $U$ on $L, U$ is said to be left-conjunctive and right-conjunctive if $U(0,1)=0$ and $U(1,0)=0$, respectively. $U$ is said to be conjunctive if both $U(1,0)=0$ and $U(1,0)=0$ since it satisfies the classical boundary conditions of AND. If $U(1,0)=1$ $(U(0,1)=1)$, then we call $U$ left-disjunctive (rightdisjunctive). We call $U$ disjunctive if both $U(1,0)=1$ and $U(0,1)=1$ by a similar reason.

If a left (right) semi-uninorm $U$ is associative, then $U$ is the left (right) uninorm [14-15] on $L$.

If a left (right) semi-uninorm $U$ with the left (right) neutral element $e_{L} \in L \quad\left(e_{R} \in L\right)$ has a right (left) neutral element $e_{R} \in L \quad\left(e_{L} \in L\right)$, then $e_{L}=U\left(e_{L}, e_{R}\right)=e_{R}$. Let $e=e_{L}=e_{R}$. Here, $U$ is the semi-uninorm [16]. In particular, if the neutral element $e=1$, then the semiuninorm $U$ becomes a $t$-seminorm [22] or a semi-copula [23-24]; if the neutral element $e=0$, then the semi- uninorm $U$ becomes a $t$-semiconorm [25].

Clearly, $U(0,0)=0$ and $U(1,1)=1$ hold for any left (right) semi-uninorm $U$ on $L$. Moreover, the left (right) neutral elements need not to be unique. In fact, the projection operator given by $U(x, y)=x$ for all $x, y \in L$ is such that any element in $L$ is a right neutral element. But, left (right) neutral elements are all idempotent [26] because $U\left(e_{L}, e_{L}\right)=e_{L} \quad\left(U\left(e_{R}, e_{R}\right)=e_{R}\right)$ for any left (right) neutral element $e_{L} \quad\left(e_{R}\right)$ of $U$.

Definition 2.2 (Wang and Fang [15]). A binary operation $U$ on $L$ is called left (right) $\vee$-distributive if

$$
\begin{gathered}
U\left(\vee_{j \in J} x_{j}, y\right)=\vee_{j \in J} U\left(x_{j}, y\right) \quad \forall x_{j}, y \in L \\
\left(U\left(x, \vee_{j \in J} y_{j}\right)=\vee_{j \in J} U\left(x, y_{j}\right) \quad \forall x, y_{j} \in L\right) ;
\end{gathered}
$$

left (right) $\wedge$-distributive if

$$
\begin{array}{cc}
U\left(\wedge_{j \in J} x_{j}, y\right)=\wedge_{j \in J} U\left(x_{j}, y\right) & \forall x_{j}, y \in L \\
\left(U\left(x, \wedge_{j \in J} y_{j}\right)=\wedge_{j \in J} U\left(x, y_{j}\right)\right. & \left.\forall x, y_{j} \in L\right) .
\end{array}
$$

If a binary operation $U$ is left $\vee$-distributive $(\wedge-$ distributive) and also right $\vee$-distributive $(\wedge$-distributive), then $U$ is said to be $\vee$-distributive $(\wedge$-distributive).

Noting that the least upper bound of the empty set is 0 and the greatest lower bound of the empty set is 1 , we have

$$
\begin{gathered}
U(0, y)=U\left(\vee_{j \in \Phi} x_{j}, y\right)=\vee_{j \in \Phi} U\left(x_{j}, y\right)=0 \\
\left.U(x, 0)=U\left(x, \vee_{j \in \Phi} y_{j}\right)=\vee_{j \in \Phi} U\left(x, y_{j}\right)=0\right)
\end{gathered}
$$

for any $x, y \in L$ when $U$ is left (right) $\vee$-distributive,

$$
\begin{gathered}
U(1, y)=U\left(\wedge_{j \in \Phi} x_{j}, y\right)=\wedge_{j \in \Phi} U\left(x_{j}, y\right)=1 \\
\left(U(x, 1)=U\left(x, \wedge_{j \in \Phi} y_{j}\right)=\wedge_{j \in \Phi} U\left(x, y_{j}\right)=1\right)
\end{gathered}
$$

for any $x, y \in L$ when $U$ is left (right) $\wedge$-distributive.

For the sake of convenience, we introduce the following symbols:

$U_{S}^{e_{L}}(L)\left(U_{s}^{e_{R}}(L)\right)$ : the set of all left (right) semi-uninorms with the left (right) neutral element $e_{L}\left(e_{R}\right)$ on $L$;

$U_{s \vee}^{e_{L}}(L)\left(U_{s \vee}^{e_{R}}(L)\right)$ : the set of all right $\vee$-distributive left (right) semi-uninorms with the left (right) neutral element $e_{L}\left(e_{R}\right)$ on $L$;

$U_{\vee S}^{e_{L}}(L)\left(U_{\vee S}^{e_{R}}(L)\right)$ : the set of all left $\vee$-distributive left (right) semi-uninorms with the left (right) neutral element $e_{L}\left(e_{R}\right)$ on $L$.

Now, we present two examples of left and right semiuninorms on $L$.

Example 2.1 ( $\mathrm{Su}$ et al. [17]). Let $e_{L} \in L$, 


$$
\begin{gathered}
U_{s W}^{e_{L}}(x, y)=\left\{\begin{array}{l}
y \text { if } x \geq e_{L}, \\
0 \text { otherwise, }
\end{array} U_{s M}^{e_{L}}(x, y)=\left\{\begin{array}{l}
y \text { if } x \geq e_{L}, \\
1 \text { otherwise }
\end{array}\right.\right. \\
U_{s M}^{e_{L}^{*}}(x, y)=\left\{\begin{array}{cc}
0 & \text { if } y=0 \\
y & \text { if } x \leq e_{L}, y \neq 0 \\
1 & \text { otherwise }
\end{array}\right.
\end{gathered}
$$

where $x$ and $y$ are elements of $L$. Then $U_{s W}^{e_{L}}$ and $U_{s M}^{e_{L}}$ are, respectively, the smallest and greatest elements of $U_{s}^{e_{L}}(L) ; U_{s W}^{e_{L}}$ and $U_{s M}^{e_{L}^{*}}$ are, respectively, the smallest and greatest elements of $U_{s \vee}^{e_{L}}(L)$.

Example 2.2 (Su et al. [17]). Let $e_{R} \in L$,

$$
\begin{gathered}
U_{s W}^{e_{R}}(x, y)=\left\{\begin{array}{l}
x \text { if } y \geq e_{R}, \\
0 \text { otherwise, }
\end{array} \quad U_{s M}^{e_{R}}(x, y)=\left\{\begin{array}{l}
x \text { if } y \geq e_{R}, \\
1 \text { otherwise }
\end{array}\right.\right. \\
U_{s M}^{e_{R}^{*}}(x, y)= \begin{cases}0 & \text { if } x=0, \\
x & \text { if } y \leq e_{L}, x \neq 0, \\
1 & \text { otherwise }\end{cases}
\end{gathered}
$$

where $x$ and $y$ are elements of $L$. Then $U_{s W}^{e_{R}}$ and $U_{S M}^{e_{R}}$ are, respectively, the smallest and greatest elements of $U_{s}^{e_{R}}(L) ; U_{s W}^{e_{R}}$ and $U_{s M}^{e_{R}^{*}}$ are, respectively, the smallest and greatest elements of $U_{\vee S}^{e_{R}}(L)$.

\section{The Residual Operations of Left and Right Semi-Uninorms}

Recently, De Baets and Fodor [18] investigated the residual operators of uninorms on $[0,1]$, Torrens et al. [1920] studied the implications and coimplications derived from uninorms on [0,1], Wang and Fang [14] discussed the residual implications of left and right uninorms on a complete lattice, and Liu [16] researched semi-uninorms and implications on a complete lattice. In this section, based on $[14,16,18-20]$, we consider the residual implications of left and right semi-uninorms on a complete lattice.

First of all, we recall the definition of implications.

Definition 3.1 (De Baets and Fodor [18], Baczynski and Jayaram [27], De Baets [28]). An implication I on $L$ is a hybrid monotonous (with non-increasing first and non-decreasing second partial mappings) binary operation that satisfies the corner conditions $I(0,0)=I(1,1)=1$ and $I(1,0)=0$.

Implications are extensions of the Boolean implication $\rightarrow(P \rightarrow Q$ meaning that $P$ is sufficient for $Q)$.

Note that for any implication $I$ on $L$, due to the monotonicity, the absorption principle holds, i.e., $I(0, x)=I(x, 1)=1$ for any $x \in L$.
We denote the set of all implications and the set of all right $\wedge$-distributive implications on $L$ by $I(L)$ and $I_{\wedge}(L)$, respectively.

Example 3.1. Let

$$
\begin{gathered}
I_{W}(x, y)= \begin{cases}1 & \text { if } x=0 \text { or } y=1, \\
0 & \text { otherwise }\end{cases} \\
I_{M}(x, y)= \begin{cases}0 \text { if }(x, y)=(1,0), \\
1 & \text { otherwise },\end{cases}
\end{gathered}
$$

where $x$ and $y$ are elements of $L$. It is easy to see that $I_{W}$ and $I_{M}$ are, respectively, the smallest and greatest elements of $I(L)$ and $I_{W}$ is also the smallest element of $I_{\wedge}(L)$.

Definition 3.1. Let $U$ be a binary operation on $L$. Define $I_{U}^{L}, I_{U}^{R} \in L^{L \times L}$ as follows:

$$
\begin{array}{ll}
I_{U}^{L}(x, y)=\vee\{z \in L \mid U(z, x) \leq y\} & \forall x, y \in L, \\
I_{U}^{R}(x, y)=\vee\{z \in L \mid U(x, z) \leq y\} & \forall x, y \in L .
\end{array}
$$

Here, $I_{U}^{L}$ and $I_{U}^{R}$ are, respectively, called the left and right residual operators of $U$.

For any operation $U$ on $L$ and $x, y \in L$, it is straightforward to verify that

(1) $I_{U}^{L}(x, 1)=I_{U}^{R}(x, 1)=1$.

(2) $x \leq I_{U}^{L}(y, U(x, y))$ and $y \leq I_{U}^{R}(x, U(x, y))$.

(3) If $U(1,0)=0$, then $I_{U}^{L}(0, y)=1$ and if $U(0,1)=0$, then $I_{U}^{R}(0, y)=1$.

When $U$ is a left (right) semi-uninorm on $L$, it is easy to see that $I_{U}^{L}$ and $I_{U}^{R}$ are all non-increasing in the first variable and non-decreasing in the second one.

Example 3.2. For some left and right semi-uninorms in Examples 2.1 and 2.2, a simple computation shows that

$$
\begin{gathered}
I_{U_{s W}^{e_{R}}}^{L}(x, y)=\left\{\begin{array}{l}
y \text { if } x \geq e_{R}, \\
1 \text { otherwise, }
\end{array} \quad I_{U_{s W}^{e_{L}}}^{R}(x, y)=\left\{\begin{array}{l}
y \text { if } x \geq e_{L}, \\
1 \text { otherwise }
\end{array}\right.\right. \\
I_{U_{s M}^{e_{R}}}^{L}(x, y)=I_{U_{s M}^{e_{R}}}^{L}(x, y)=\left\{\begin{array}{l}
1 \text { if } y=1, \\
y \text { if } x \geq e_{R}, \\
0 \text { otherwise, }
\end{array}\right. \\
I_{U_{s M}^{e_{L}}}^{L}(x, y)=\left\{\begin{array}{cc}
1 & \text { if } y=1, \\
e_{L} & \text { if } x \leq y<1, \\
0 & \text { otherwise, }
\end{array}\right.
\end{gathered}
$$




$$
\begin{aligned}
& I_{U_{s M}^{e_{L}}}^{L}(x, y)=\left\{\begin{array}{c}
1 \text { if } x=0 \text { or } y=1 \\
e_{L} \text { if } 0<x \leq y<1 \\
0 \quad \text { otherwise }
\end{array}\right. \\
& I_{U_{s M}^{e_{L}}}^{R}(x, y)=I_{U_{s M}^{e_{L}}{ }^{*}}^{R}(x, y)=\left\{\begin{array}{l}
1 \text { if } y=1, \\
y \text { if } x \leq e_{L}, \\
0 \text { otherwise, }
\end{array}\right. \\
& I_{U_{s M}^{e_{R}}}^{R}(x, y)=\left\{\begin{array}{c}
1 \quad \text { if } y=1 \\
e_{R} \text { if } x \leq y<1 \\
0 \quad \text { otherwise }
\end{array}\right. \\
& I_{U_{s M}^{e R}}^{R}(x, y)=\left\{\begin{array}{l}
1 \text { if } x=0 \text { or } y=1, \\
e_{R} \text { if } 0<x \leq y<1, \\
0 \quad \text { otherwise. }
\end{array}\right.
\end{aligned}
$$

When $e_{L}, e_{R} \in L \backslash\{0,1\}$, we see that $I_{U_{s M}^{e_{L}}}^{L}$ and $I_{U_{s M}^{e_{R}}}^{R}$ are two implications, $I_{U_{s W}^{e}}^{L}$ and $I_{U_{s W}^{e_{W}}}^{R}$ are two right $\wedge$ -distributive implications, but $I_{U_{s M}^{e_{R}}}^{L}, I_{U_{s M}^{e_{R}^{*}}}^{L}, I_{U_{s M}^{e_{L}}}^{L}, I_{U_{s M}^{e_{L}}}^{R}$, $I_{U_{s M}^{e_{L}} *}^{R}$ and $I_{U_{s M}^{e_{R}}}^{R}$ are not implications.

Theorem 3.1. Let $U \in U_{s}^{e_{L}}(L)$.

(1) For any $x, y \in L, x \leq y \Rightarrow I_{U}^{L}(x, y) \geq e_{L}$.

(2) $I_{U}^{R}$ satisfies the neutrality principle with $e_{L}$, i.e., $I_{U}^{R}\left(e_{L}, y\right)=y$ for any $y \in L$.

(3) If $U$ is left-conjunctive, then $I_{U}^{R} \in I(L)$.

(4) If $U \in U_{S \vee}^{e_{L}}(L)$ is left-conjunctive, then

$I_{U}^{R} \in I_{\wedge}(L)$ and $I_{U}^{R}(x, y)=\max \{z \in L \mid U(x, z) \leq y\}$.

Here, $I_{U}^{R}$ is called the right residual implication of the left semi-uninorm $U$.

Proof. Clearly, statements (1) and (2) hold.

(3) If $U$ is left-conjunctive, then $U(0,1)=0$ and

$$
I_{U}^{R}(0,0)=\vee\{z \in L \mid U(0, z)=0\}=1 .
$$

By the non-decreasingness of $U$, we see that

$$
\begin{aligned}
& I_{U}^{R}(1,0)=\vee\{z \in L \mid U(1, z)=0\} \\
& \leq \vee\left\{z \in L \mid z=U\left(e_{L}, z\right) \leq U(1, z)=0\right\}=0 .
\end{aligned}
$$

Moreover, it follows from the statements before Example 3.2 that $I_{U}^{R}(1,1)=1$ and $I_{U}^{R}$ is non-increasing in its first and non-decreasing in its second variable. Therefore, $I_{U}^{R}$ is an implication on $L$.
(4) Assume that $U$ is left-conjunctive right $\vee$-distributive. Then, $I_{U}^{R} \in I(L)$ by statement (3). Let $x, y, z \in L$. If $U(x, z) \leq y$, then $z \leq I_{U}^{R}(x, y)$; if $z \leq I_{U}^{R}(x, y)$, then it follows from the non-decreasingness of $U$ that

$$
\begin{aligned}
& U(x, z) \leq U\left(x, I_{U}^{R}(x, y)\right)=U(x, \vee\{z \in L \mid U(x, z) \leq y\} \\
= & \vee\{U(x, z) \mid z \in L, U(x, z) \leq y\} \leq y
\end{aligned}
$$

Noting that $U\left(x, I_{U}^{R}(x, y)\right) \leq y$, we know that

$$
I_{U}^{R}(x, y)=\max \{z \in L \mid U(x, z) \leq y\}
$$

Moreover, when $J \neq \Phi$, for any $x, y_{j} \in L(j \in J)$, we have that

$$
\begin{aligned}
& I_{U}^{R}\left(x, \wedge_{j \in J} y_{j}\right)=\vee\left\{z \in L \mid U(x, z) \leq \wedge_{j \in J} y_{j}\right\} \\
= & \vee\left\{z \in L \mid U(x, z) \leq y_{j} \forall j \in J\right\} \\
= & \vee\left\{z \in L \mid z \leq I_{U}^{R}\left(x, y_{j}\right) \forall j \in J\right\} \\
= & \vee\left\{z \in L \mid z \leq \wedge_{j \in J} I_{U}^{R}\left(x, y_{j}\right)\right\}=\wedge_{j \in J} I_{U}^{R}\left(x, y_{j}\right) .
\end{aligned}
$$

When $J=\Phi$, we see that

$$
I_{U}^{R}\left(x, \wedge_{j \in \Phi} y_{j}\right)=I_{U}^{R}(x, 1)=1=\wedge_{j \in \Phi} I_{U}^{R}\left(x, y_{j}\right) .
$$

Therefore, $I_{U}^{R}$ is right $\wedge$-distributive, i.e., $I_{U}^{R} \in I_{\wedge}(L)$. The theorem is proved.

When $e_{L}<1$, for the right $\vee$-distributive left semiuninorm $U_{s M}^{e_{L}^{*}}$, we see that $I_{U_{s M}^{e_{L}}{ }^{*}}^{L}$ by Example 3.2, but $I_{U_{s M}^{e_{L}}{ }^{*}}^{L}\left(e_{L}, y\right)=e_{L} \neq y$ when $e_{L}<y<1$, i.e., $I_{U_{s M}^{e_{L}}{ }^{*}}^{L}$ does not satisfy the neutrality principle with $e_{L}$. This illustrates Theorem 3.1 doesn't hold for the left residual operator of a left semi-uninorm.

If $P$ and $Q$ are two propositions, then the generalized modus ponens (GMP) [18] gives a lower bound for the truth value of $Q$ when the truth values of propositions $P$ and $P \rightarrow Q$ are known. By the proof of Theorem 3.1(4), we know that $U$ and $I_{U}^{R}$ satisfy the GMP rule:

$$
U\left(x, I_{U}^{R}(x, y)\right) \leq y \forall x, y \in L
$$

and the following right residual principle:

$$
\left.U(x, z) \leq y \Leftrightarrow z \leq I_{U}^{R}(x, y)\right) \forall x, y, z \in L
$$

when a binary operation $U$ is right $\vee$-distributive.

Similarly, $U$ and $I_{U}^{L}$ satisfy GMP rule in the form: 


$$
U\left(I_{U}^{L}(x, y), x\right) \leq y \forall x, y \in L
$$

and the following left residual principle:

$$
\left.U(z, x) \leq y \Leftrightarrow z \leq I_{U}^{L}(x, y)\right) \forall x, y, z \in L
$$

when $U$ is left $\vee$-distributive. Thus, for right semiuninorms on $L$, we have a similar result.

Theorem 3.2. Let $U \in U_{S}^{e_{R}}(L)$.

(1) For any $x, y \in L, \quad x \leq y \Rightarrow I_{U}^{R}(x, y) \geq e_{R}$.

(2) $I_{U}^{L}$ satisfies the neutrality principle with $e_{R}$, i.e., $I_{U}^{L}\left(e_{R}, y\right)=y$ for any $y \in L$.

(3) If $U$ is right-conjunctive, then $I_{U}^{L} \in I(L)$.

(4) If $U \in U_{\vee S}^{e_{R}}(L)$ is right-conjunctive, then

$$
I_{U}^{L} \in I_{\wedge}(L) \text { and } I_{U}^{L}(x, y)=\max \{z \in L \mid U(z, x) \leq y\} .
$$

Here, $I_{U}^{L}$ is called the left residual implication of the left semi-uninorm $U$.

Combining Theorems 3.1 and 3.2, we know that both $I_{U}^{L}$ and $I_{U}^{R}$ are all right $\vee$-distributive implications when $U$ is a $\vee$-distributive left (right) semi-uninorm.

Theorem 3.3. (1) If $U \in U_{\vee S}^{e_{L}}(L)$, then $I_{U}^{L}$ is right $\wedge$ -distributive and satisfies the left residual principle and the order property with $e_{L}$ :

$$
x \leq y \Leftrightarrow I_{U}^{L}(x, y) \geq e_{L} \forall x, y \in L .
$$

(2) If $U \in U_{s \vee}^{e_{R}}(L)$, then $I_{U}^{R}$ is right $\wedge$-distributive and satisfies the right residual principle and the order property with $e_{R}$ :

$$
x \leq y \Leftrightarrow I_{U}^{R}(x, y) \geq e_{R} \forall x, y \in L .
$$

Proof. Assume that $U$ is a left $\wedge$-distributive left semi-uninorms with the left neutral element $e_{L}$. By virtue of the proof of Theorem 3.1(4), we can see that $I_{U}^{L}$ is right $\wedge$-distributive and satisfies the left residual principle. Moreover, if $x, y \in L$ and $x \leq y$, then it follows from Theorem 3.1(1) that $I_{U}^{L}(x, y) \geq e_{L}$; if $I_{U}^{L}(x, y) \geq e_{L}$, then

$$
x=U\left(e_{L}, x\right) \leq U\left(I_{U}^{L}(x, y), x\right) \leq y .
$$

Thus, $I_{U}^{L}$ satisfies the order property with $e_{L}$.

Similarly, we can show that $I_{U}^{R}$ is right $\wedge$-distributive and satisfies the right residual principle and the order property with $e_{R}$ when $U$ is a right $\vee$-distributive right semi-uninorms with the right neutral element
$e_{R}$

The theorem is proved.

In particular, if $U$ is a $\vee$-distributive semi-uninorm with the neutral element $e$, then $I_{U}^{L}$ and $I_{U}^{R}$ satisfy the the residual principle (RP) and the order property (OP) and are all right $\wedge$-distributive implications (see Theorem 3.6 in [16]).

\section{The Left and Right Semi-Uninorms Induced by Implications}

Liu [16] discussed the semi-uninorms induced by implications and $\mathrm{Su}$ and Wang [29] studied the pseudouninorms induced by coimplications. In this section, based on these works, we investigate the left and right semiuninorms induced by implications on a complete lattice.

Definition 4.1. Let $I$ be a binary operation on $L$. Define two induced operators $U_{I}^{L}$ and $U_{I}^{R}$ of $I$ as follows:

$$
\begin{array}{cc}
U_{I}^{L}(x, y)=\wedge\{z \in L \mid x \leq I(y, z)\} & \forall x, y \in L, \\
U_{I}^{R}(x, y)=\wedge\{z \in L \mid y \leq I(y, z)\} & \forall x, y \in L .
\end{array}
$$

Clearly, $U_{I}^{L}(0, x)=U_{I}^{R}(x, 0)=0, U_{I}^{L}(1, x)=U_{I}^{R}(x, 1)$ for any $x \in L$, and $U_{I}^{L}=U_{I}^{R}$ if $I$ satisfies the condition:

$$
x \leq I(y, z) \Leftrightarrow y \leq I(x, z) \forall x, y, z \in L .
$$

When $I$ is hybird monotonous, it is easy to see that $U_{I}^{L}$ and $U_{I}^{R}$ are all non-decreasing in its each variable. Moreover, for any binary operation $I$, it follows from Definition 4.1 that

$$
U_{I}^{L}(I(x, y), x) \leq y, U_{I}^{R}(x, I(x, y)) \leq y \quad \forall x, y \in L .
$$

These explain that $U_{I}^{L}$ and $I, U_{I}^{R}$ and $I$ satisfy the GMP rule.

Example 4.1. For two implications $I_{W}$ and $I_{M}$ in Example 3.1, we have that

$$
\begin{aligned}
& U_{I_{W}}^{L}(x, y)=U_{I_{W}}^{R}(x, y)=\left\{\begin{array}{lr}
1 & \text { if } x=0 \text { or } y=0, \\
0 & \text { otherwise, }
\end{array}\right. \\
& U_{I_{M}}^{L}(x, y)=\left\{\begin{array}{lr}
\wedge_{a \in L \backslash\{0\}} & \text { if } x>0 \text { and } y=1, \\
0 & \text { otherwise, }
\end{array}\right. \\
& U_{I_{M}}^{R}(x, y)=\left\{\begin{array}{lr}
\wedge_{a \in L \backslash\{0\}} & \text { if } x=1 \text { and } y>0, \\
0 & \text { otherwise. }
\end{array}\right.
\end{aligned}
$$


Thus, four operations induced by implications $I_{W}$ and $I_{M}$ are neither left semi-uninorms nor right semiuninorms on $L$.

Below, we find some conditions such that these operations induced by implications are left or right semiuninorms.

Theorem 4.1. Let $I$ be an implication on $L$.

(1) If $I$ satisfies the order property with $e_{L}$, then $U_{I}^{L} \in U_{s}^{e_{L}}(L)$; if $I$ satisfies the neutrality principle with $e_{L}$, then $U_{I}^{R} \in U_{s}^{e_{L}}(L)$. Here, $U_{I}^{L}$ and $U_{I}^{R}$ are called the left semi-uninorms induced by the implication $I$.

(2) If I satisfies the order property with $e_{R}$, then $U_{I}^{R} \in U_{S}^{e_{R}}(L)$; if $I$ satisfies the neutrality principle with $e_{R}$, then $U_{I}^{L} \in U_{s}^{e_{R}}(L)$. Here, $U_{I}^{L}$ and $U_{I}^{R}$ are called the right semi-uninorms induced by the implication $I$.

(3) If I satisfies the order property with $e_{L}$ and the neutrality principle with $e_{R}$, then $U_{I}^{L}$ is a semi-uninorm on $L$.

(4) If I satisfies the order property with $e_{R}$ and the neutrality principle with $e_{L}$, then $U_{I}^{R}$ is also a semi-uninorm on $L$.

Proof. Assume that $I \in I(L)$. Then $U_{I}^{L}$ is nondecreasing in each variable. If $I$ satisfies the order property with $e_{L}$, then

$$
\begin{aligned}
& U_{I}^{L}\left(e_{L}, y\right)=\wedge\left\{z \in L \mid e_{L} \leq I(y, z)\right\} \\
& =\wedge\{z \in L \mid y \leq z\}=y \quad \forall y \in L .
\end{aligned}
$$

Thus, $U_{I}^{L} \in U_{S}^{e_{L}}(L)$. If $I$ satisfies the neutrality principle with $e_{L}$, then

$$
\begin{aligned}
& U_{I}^{R}\left(e_{L}, y\right)=\wedge\left\{z \in L \mid y \leq I\left(e_{L}, z\right)\right\} \\
& =\wedge\{z \in L \mid y \leq z\}=y \quad \forall y \in L .
\end{aligned}
$$

So, $U_{I}^{R} \in U_{S}^{e_{L}}(L)$.

Similarly, we can show that $U_{I}^{R} \in U_{s}^{e_{R}}(L)$ when the implication $I$ satisfies the order property with $e_{R}$ and $U_{I}^{L} \in U_{S}^{e_{R}}(L)$ when $I$ satisfies the neutrality principle with $e_{R}$.

If $I$ satisfies the order property with $e_{L}$ and the neutrality principle with $e_{R}$, then

$$
U_{I}^{L}\left(e_{L}, x\right)=U_{I}^{L}\left(x, e_{R}\right)=x \quad \forall x \in L .
$$

Thus, $e_{L}=e_{R}$, i.e., $U_{I}^{L}$ is a semi-uninorm with the neutral element $e_{L}\left(=e_{R}\right)$ on $L$.

In a similar way, we can see that $U_{I}^{R}$ is also a semi-uninorm on $L$ when $I$ satisfies the order property with $e_{R}$ and the neutrality principle with $e_{L}$.

The theorem is proved.

When $I \in I(L), I(0, x)=1$ for any $x \in L$ and hence it follows from Definition 4.1 that

$$
U_{I}^{L}(1,0)=U_{I}^{R}(0,1) .
$$

Thus, $U_{I}^{L}$ and $U_{I}^{R}$ in Theorem 4.1 are all conjunctive left or right semi-uninorms induced by the implication $I$.

Theorem 4.2. Let $I \in I_{\wedge}(L)$.

(1) If I satisfies the order property with $e_{L}$, then $U_{I}^{L} \in U_{\mathrm{V} S}^{e_{L}}(L) ;$ if $I$ satisfies the neutrality principle with $e_{L}$, then $U_{I}^{R} \in U_{s \vee}^{e_{L}}(L)$.

(2) If I satisfies the order property with $e_{R}$, then $U_{I}^{R} \in U_{s \vee}^{e_{R}}(L) ;$ if $I$ satisfies the neutrality principle with $e_{R}$, then $U_{I}^{L} \in U_{\vee S}^{e_{R}}(L)$.

(3) If I satisfies the order property with $e_{L}$ and the neutrality principle with $e_{R}$, then $U_{I}^{L}$ is a left $\vee$ -distributive semi-uninorm on $L$.

(4) If I satisfies thet order property with $e_{R}$ and the neutrality principle with $e_{L}$, then $U_{I}^{R}$ is also a right $\vee$-distributive semi-uninorm on $L$.

Proof. Assume that $I$ is a right $\wedge$-distributive implication. Let $x, y, z \in L$. If $x \leq I(y, z)$, then it follows from Definition 4.1 that $U_{I}^{L}(x, y) \leq z$; if $U_{I}^{L}(x, y) \leq z$, then

$$
\begin{aligned}
& I(y, z) \geq I\left(y, U_{I}^{L}(x, y)\right) \\
& =I(y, \wedge\{z \in L \mid x \leq I(y, z)\}) \\
& =\wedge\{I(y, z) \mid z \in L, x \leq I(y, z)\} \geq x .
\end{aligned}
$$

Noting that $x \leq I\left(y, U_{I}^{L}(x, y)\right)$, we know that

$$
U_{I}^{L}(x, y)=\min \{z \in L \mid x \leq I(y, z)\} .
$$

Moreover, when $J \neq \Phi$, for any $x_{j}, y \in L(j \in J)$, we have that 


$$
\begin{aligned}
U_{I}^{L}\left(\vee_{j \in J} x_{j}, y\right) & =\wedge\left\{z \in L \mid \vee_{j \in J} x_{j} \leq I(y, z)\right\} \\
& =\wedge\left\{z \in L \mid x_{j} \leq I(y, z) \forall j \in J\right\} \\
& =\wedge\left\{z \in L \mid U_{I}^{L}\left(x_{j}, y\right) \leq z \forall j \in J\right\} \\
& =\wedge\left\{z \in L \mid \vee_{j \in J} U_{I}^{L}\left(x_{j}, y\right) \leq z\right\} \\
& =\vee_{j \in J} U_{I}^{L}\left(x_{j}, y\right) .
\end{aligned}
$$

When $J=\Phi$, we see that

$$
U_{I}^{L}\left(\vee_{j \in \Phi} x_{j}, y\right)=U_{I}^{L}(0, y)=0=\vee_{j \in \Phi} U_{I}^{L}\left(x_{j}, y\right) .
$$

Thus, $U_{I}^{L}$ is left $\vee$-distributive. Therefore, by virtue of Theorem 4.1, $U_{I}^{L} \in U_{\vee S}^{e_{L}}(L)$ when $I$ satisfies the order property with $e_{L}$ and $U_{I}^{L} \in U_{\vee S}^{e_{R}}(L)$ when $I$ satisfies the neutrality principle with $e_{R}$.

Similarly, we can show that $U_{I}^{R}$ is a right $\vee$-distributive right semi-uninorm and left semi-uninorm when $I$ satisfies the order property with $e_{R}$ and the neutrality principle with $e_{L}$, respectively.

Statements (3) and (4) are the direct consequences of statements (1) and (2) and Theorem 4.1.

The theorem is proved.

By virtue of Theorem 4.2, we see that $U_{I}^{L}$ and $U_{I}^{R}$ are a left $\vee$-distributive semi-uninorm and a right $\vee$ -distributive semi-uninorm on $L$ when $I \in I_{\wedge}(L)$ satisfies the order property (OP) and neutrality principle (NP), respectively. This explains Theorem 4.2 is a generalization of Theorem 4.5 in [16].

When $I$ is a right $\wedge$-distributive implication on $L$, by the proof of Theorem 4.2, we know that $I, U_{I}^{L}$ and $U_{I}^{R} \quad$ satisfy the following adjunction conditions:

$$
\begin{aligned}
& x \leq I(y, z) \Leftrightarrow U_{I}^{L}(x, y) \leq z, \\
& y \leq I(x, z) \Leftrightarrow U_{I}^{R}(x, y) \leq z \forall x, y, z \in L .
\end{aligned}
$$

Moreover, we have

$$
U_{I}^{R}(x, y)=\min \{z \in L \mid y \leq I(x, z)\} .
$$

\section{The Relationships between Left (Right) Semi-Uninorms and Implications}

In the final section, we reveal the relationships between conjunctive right (left) $\vee$-distributive left (right) semiuninorms and right $\wedge$-distributive implications which satisfy the neutrality principle on a complete lattice.

Theorem 5.1.
(1) If $U \in U_{\vee S}^{e_{R}}(L)$ is right-conjunctive, then $I_{U}^{L} \in I_{\wedge}(L)$ satisfies the neutrality principle with $e_{R}$ and $U_{I_{U}^{L}}^{L}=U$.

(2) If $U \in U_{s \vee}^{e_{L}}(L)$ is left-conjunctive, then $I_{U}^{R} \in I_{\wedge}(L)$ satisfies the neutrality principle with $e_{L}$ and $U_{I_{U}^{R}}^{R}=U$.

(3) If $I \in I_{\wedge}(L)$ satisfies the neutrality principle with $e_{L}$, then $U_{I}^{R} \in U_{s \vee}^{e_{L}}(L)$ is conjunctive and $I_{U_{I}^{R}}^{R}=I$.

(4) If $I \in I_{\wedge}(L)$ satisfies the neutrality principle with $e_{R}$, then $U_{I}^{L} \in U_{s \vee}^{e_{R}}(L)$ is conjunctive and $I_{U_{I}^{L}}^{L}=I$.

Proof. We only prove the statements (1) and (3) hold.

(1) If $U$ is a right-conjunctive left $\vee$-distributive right semi-uninorm, then $I_{U}^{L} \in I_{\wedge}(L)$ and satisfies the neutrality principle with $e_{R}$ by Theorem 3.2. Moreover, it follows from the left residual principle that

$$
\begin{aligned}
& U_{I_{U}^{L}}^{L}(x, y)=\wedge\left\{z \in L \mid x \leq I_{U}^{L}(y, z)\right\} \\
& =\wedge\{z \in L \mid U(x, y) \leq z\}=U(x, y) \quad \forall x, y \in L .
\end{aligned}
$$

Thus, $U_{I_{U}^{L}}^{L}=U$.

(3) If $I \in I_{\wedge}(L)$ satisfies the neutrality principle with $e_{L}$, then $U_{I}^{R}$ is a conjunctive right $\vee$-distributive left semi-uninorm by Theorem 4.2. Moreover, it follows from the adjunction conditions that

$$
\begin{aligned}
& I_{U_{I}^{R}}^{R}(x, y)=\vee\left\{z \in L \mid U_{I}^{R}(y, z) \leq y\right\} \\
& =\vee\{z \in L \mid z \leq I(x, y)\}=I(x, y) \quad \forall x, y \in L .
\end{aligned}
$$

Therefore, $I_{U_{I}^{R}}^{R}=I$.

The theorem is proved.

We denote by $U_{c s v}^{e_{L}}(L)$ and $U_{v c s}^{e_{R}}(L)$, respectively, the set of all conjunctive right $\vee$-distributive left semiuninorms and the set of all conjunctive left $\vee$-distributive right semi-uninorms; by $I_{\wedge}^{e_{L}}(L)$ and $I_{\wedge}^{e_{R}}(L)$, respectively, the set of all right $\wedge$-distributive implications which satisfy the neutrality principle with $e_{L}$ and $e_{R}$ on a complete lattice.

It is easy to verify that $U_{c s \vee}^{e_{L}}(L)$ and $U_{v c S}^{e_{R}}(L)$ are two join-semilattices with the smallest elemens $U_{s W}^{e_{L}}$ and 
$U_{s W}^{e_{R}}$, respectively; and $I_{\wedge}^{e_{L}}(L)$ and $I_{\wedge}^{e_{R}}(L)$ are two meetsemilattices with the greatest elements $I_{U_{s W}^{e L}}^{R}$ and $I_{U_{s W}^{e R}}^{L}$, respectively, where

$$
\begin{aligned}
& \left(U_{1} \vee U_{2}\right)(x, y)=U_{1}(x, y) \vee U_{2}(x, y), \\
& \left(I_{1} \wedge I_{2}\right)(x, y)=I_{1}(x, y) \wedge I_{2}(x, y) \forall x, y \in L .
\end{aligned}
$$

Define two mappings $\quad \phi: U_{c s \vee}^{e_{L}}(L) \rightarrow I_{\wedge}^{e_{L}}(L) \quad$ and $\varphi: U_{\vee c S}^{e_{R}}(L) \rightarrow I_{\wedge}^{e_{R}}(L)$ as follows:

$$
\phi(U)=I_{U}^{R} \forall U \in U_{c s \vee}^{e_{L}}(L), \varphi(U)=I_{U}^{L} \forall U \in U_{\vee c s}^{e_{R}}(L) .
$$

Then it follows from Theorem 5.1 that $\phi$ and $\varphi$ are all invertible and

$$
\phi^{-1}(I)=U_{I}^{R} \forall I \in I_{\wedge}^{e_{L}}(L), \varphi^{-1}(I)=U_{I}^{L} \forall I \in I_{\wedge}^{e_{L R}}(L) .
$$

Moreover, we have the following theorem.

Theorem 5.2.

(1) If $U_{1}, U_{2} \in U_{c s \vee}^{e_{L}}(L)$, then $I_{U_{1} \vee U_{2}}^{R}=I_{U_{1}}^{R} \wedge I_{U_{2}}^{R}$.

(2) If $U_{1}, U_{2} \in U_{\vee c s}^{e_{R}}(L)$, then $I_{U_{1} \vee U_{2}}^{L}=I_{U_{1}}^{L} \wedge I_{U_{2}}^{L}$.

(3) If $I_{1}, I_{2} \in I_{\wedge}^{e_{L}}(L)$, then $U_{I_{1} \wedge I_{2}}^{R}=U_{I_{1}}^{R} \vee U_{I_{2}}^{R}$.

(4) If $I_{1}, I_{2} \in I_{\wedge}^{e_{R}}(L)$, then $U_{I_{1} \wedge I_{2}}^{L}=U_{I_{1}}^{L} \vee U_{I_{2}}^{L}$.

Proof. We only prove the statements (2) and (4) hold.

(2) If $U_{1}, U_{2} \in U_{\vee c S}^{e_{R}}(L)$, then it follows from the left residual principle that

$$
\begin{aligned}
& I_{U_{1} \vee U_{2}}^{L}(x, y)=\vee\left\{z \in L \mid\left(U_{1} \vee U_{2}\right)(z, x) \leq y\right\} \\
& =\vee\left\{z \in L \mid U_{1}(z, x) \vee U_{2}(z, x) \leq y\right\} \\
& =\vee\left\{z \in L \mid U_{1}(z, x) \leq y, U_{2}(z, x) \leq y\right\} \\
& =\vee\left\{z \in L \mid z \leq I_{U_{1}}^{L}(x, y), z \leq I_{U_{2}}^{L}(x, y)\right\} \\
& =\vee\left\{z \in L \mid z \leq I_{U_{1}}^{L}(x, y) \wedge I_{U_{2}}^{L}(x, y)\right\} \\
& =\left(I_{U_{1}}^{L} \wedge I_{U_{2}}^{L}\right)(x, y) \forall x, y \in L,
\end{aligned}
$$

i.e., $I_{U_{1} \vee U_{2}}^{L}=I_{U_{1}}^{L} \wedge I_{U_{2}}^{L}$.

(4) If $I_{1}, I_{2} \in I_{\wedge}^{e_{R}}(L)$, then it follows from the adjunction condition that

$$
\begin{aligned}
& U_{I_{1} \wedge I_{2}}^{L}(x, y)=\wedge\left\{z \in L \mid x \leq\left(I_{1} \wedge I_{2}\right)(y, z)\right\} \\
& =\wedge\left\{z \in L \mid x \leq I_{1}(y, z) \wedge I_{2}(y, z)\right\} \\
& =\wedge\left\{z \in L \mid x \leq I_{1}(y, z), x \leq I_{2}(y, z)\right\} \\
& =\wedge\left\{z \in L \mid U_{I_{1}}^{L}(x, y) \leq z, U_{I_{2}}^{L}(x, y) \leq z\right\} \\
& =\wedge\left\{z \in L \mid U_{I_{1}}^{L}(x, y) \vee U_{I_{2}}^{L}(x, y) \leq z\right\} \\
& =\left(U_{I_{1}}^{L} \vee U_{I_{2}}^{L}\right)(x, y) \forall x, y \in L,
\end{aligned}
$$

Therefore, $U_{I_{1} \wedge I_{2}}^{L}=U_{I_{1}}^{L} \vee U_{I_{2}}^{L}$.

The theorem is proved.

By virtue of Theorem 5.2, we know that $\phi$ and $\varphi$ are, respectively, anti-order isomorphisms of $U_{c s \vee}^{e_{L}}(L)$ onto $I_{\wedge}^{e_{L}}(L)$ and $U_{\vee c S}^{e_{R}}(L)$ onto $I_{\wedge}^{e_{R}}(L) ; \phi^{-1}$ and $\varphi^{-1}$ are, respectively, anti-order isomorphisms of $I_{\wedge}^{e_{L}}(L)$ onto $U_{c S \vee}^{e_{L}}(L)$ and $I_{\wedge}^{e_{R}}(L)$ onto $U_{\vee c s}^{e_{R}}(L)$.

\section{Conclusions and Future Works}

In this paper, we discuss the residual operations of left and right semi-uninorms and the left and right semi-uninorms induced by implications, show that the right (left) residual operator of a conjunctive right (left) $\vee$ distributive left (right) semi-uninorm is a right $\wedge$ distributive implication which satisfies the neutrality principle, give some conditions such that the operations induced by an implication constitute left or right semi-uninorms, demonstrate that the operations induced by a right $\wedge$-distributive implication, which satisfies the order property or the neutrality principle, are left (right) $\vee$ distributive left (right) semi-uninorms or right (left) semi-uninorms, and reveal the relationships between conjunctive right (left) $\vee$-distributive left (right) semiuninorms and right $\wedge$-distributive implications which satisfy the neutrality principle.

In a forthcoming paper, we will investigate the relationships between left (right) semi-uninorms and coimplications on a complete lattice.

\section{Acknowledgements}

This work is supported by the National Natural Science Foundation of China (61379064), Jiangsu Provincial Natural Science Foundation of China (BK2012672) and Science Foundation of Yancheng Teachers University (13YSYJB0108).

\section{References}

[1] E. P. Klement, R. Mesiar, and E. Pap, "Triangular Norms", Trends in Logic-Studia Logica Library, Vol. 8, Kluwer Academic Publishers, Dordrecht, 2000. 
[2] P. Flondor, G. Georgescu, and A. orgulescu, "Pseudo-t-norms and pseudo- $B L$-algebras", Soft Computing, 5, 355-371, 2001.

[3] J. Fdor and T. Keresztfalvi, "Nonstandard conjunctions and implications in fuzzy logic", International Journal of Approximate Reasoning, 12, 69-84, 1995.

[4] J. Fdor, "Srict preference relations based on weak $t$-norms", Fuzzy Sets and Systems, 43, 327-336, 1991.

[5] Z. D. Wang and Y. D. Yu, "Pseudo-t-norms and implication operators on a complete Brouwerian lattice", Fuzzy Sets and Systems, 132, 113-124, 2002.

[6] R. R. Yager and A. Rybalov, "Uninorm aggregation operators", Fuzzy Sets and Systems, 80, 111-120, 1996.

[7] J. Fodor, R. R. Yager, and A. Rybalov, "Structure of uninorms", Internat. J. Uncertainly, Fuzziness and Knowledge-Based Systems, 5, 411-427, 1997.

[8] D. Gabbay and G. Metcalfe, "fuzzy logics based on [0,1)-continuous uninorms", Arch. Math. Logic, 46, 425-449, 2007.

[9] A. K. Tsadiras and K. G. Margaritis, "The MYCIN certainty factor handling function as uninorm operator and its use as a threshold function in artificial neurons", Fuzzy Sets and Systems, 93, 263-274, 1998.

[10] R. R. Yager, "Uninorms in fuzzy system modeling", Fuzzy Sets and Systems, 122, 167-175, 2001.

[11] R. R. Yager, "Defending against strategic manipulation in uninorm-based multi-agent decision making", European J. Oper. Res., 141, 217-232, 2002.

[12] M. Mas, M. Monserrat, and J. Torrens, "On left and right uninorms", Internat. J. Uncertainly, Fuzziness and Knowledge-Based Systems, 9, 491-507, 2001.

[13] M. Mas, M. Monserrat, and J. Torrens, "On left and right uninorms on a finite chain", Fuzzy Sets and Systems, 146, 3-17, 2004.

[14] Z. D. Wang and J. X. Fang, "Residual operators of left and right uninorms on a complete lattice", Fuzzy Sets and Systems, 160, 22-31, 2009.

[15] Z. D. Wang and J. X. Fang, "Residual coimplicators of left and right uninorms on a complete lattice", Fuzzy Sets and Systems, 160, 2086-2096, 2009.

[16] H. W. Liu, "Semi-uninorm and implications on a complete lattice", Fuzzy Sets and Systems, 191, 72-82, 2012.

[17] Y. Su, Z. D. Wang, and K. M. Tang, "Left and right semi-uninorms on a complete lattice", Kybernetika, 49, 948-961, 2013.

[18] B. De Baets and J. Fodor, "Residual operators of uninorms", Soft Computing, 3, 89-100, 1999.

[19] M. Mas, M. Monserrat, and J. Torrens, "Two types of implications derived from uninorms", Fuzzy Sets and Systems, 158, 2612-2626, 2007.

[20] D. Ruiz and J. Torrens, "Residual implications and co-implications from idempotent uninorms", Kybernetika, 40, 21-38, 2004.

[21] G. Birkhoff, "Lattice Theory", American Mathematical Society Colloquium Publishers, Providence, 1967.

[22] F. Suarez Garcia and P. Gil Alvarez, "Two families of fuzzy intergrals", Fuzzy Sets and Systems, 18, 67-81, 1986.

[23] B. Bassan and F. Spizzichino, "Relations among univariate aging, bivariate aging and dependence for exchangeable lifetimes", J. Multivariate Anal., 93, 313-339, 2005.

[24] F. Durante, E. P. Klement, and R. Mesiar et al., "Conjunctors and their residual implicators: characterizations and construct methods", Mediterranean J. Math., 4, 343-356, 2007.

[25] G. De Cooman and E. E. Kerre, "Order norms on bounded partially ordered sets”, J. Fuzzy Math., 2, 281-310, 1994.

[26] B. De Baets, "Idempotent uninorms", European J. Oper. Res., 118, 631-642, 1999.

[27] M. Baczynski and B. Jayaram, "Fuzzy Implication", Studies in Fuzziness and Soft Computing, Vol. 231, Springer, Berlin, 2008.

[28] B. De Baets, "Coimplicators, the forgotten connectives", Tatra Mountains Math. Publ., 12, 229-240, 1997.

[29] Y. Su and Z. D. Wang, "Pseudo-uninorms and coimplications on a complete lattice", Fuzzy Sets and Systems, 224, 53-62, 2013. 